\title{
Non-cirrhotic portal hypertension related to azathioprine therapy in a patient with Crohn's disease
}

\author{
Jong Won Seo ${ }^{1}$, Eun Soo Kim ${ }^{1}$, Man-Hoon $\mathrm{Han}^{2}$, Young Oh Kweon ${ }^{1}$ \\ Departments of ${ }^{1}$ Internal Medicine and ${ }^{2}$ Pathology, School of Medicine, Kyungpook National University, Daegu, Korea
}

Azathioprine is widely used for the treatment of Crohn's disease (CD). Few cases from Western countries have reported idiopathic non-cirrhotic portal hypertension $(\mathrm{NCPH})$ related to thiopurine therapy in patients with inflammatory bowel disease. Idiopathic NCPH is a rare hepatic condition with intrahepatic portal hypertension but no evidence of cirrhosis or chronic liver disease. Patients with idiopathic NCPH present with symptoms of portal hypertension such as thrombocytopenia, splenomegaly and esophageal varices. We report a case of idiopathic NCPH in a 51-year-old male patient with CD who had been taking azathioprine for 5 years. He was admitted due to esophageal variceal bleeding along with splenomegaly and thrombocytopenia. Evaluation of cirrhosis or chronic liver disease showed normal-range results as estimated by FibroScan evaluation, laboratory examination for autoimmune hepatitis or viral hepatitis, and liver biopsy. This case may suggest the need for careful monitoring for manifestations of portal hypertension in Asian patients with inflammatory bowel disease receiving thiopurine treatment. (Intest Res 2021;19:247-251)

Key Words: Crohn disease; Azathioprine; Non-cirrhotic portal hypertension

\section{INTRODUCTION}

Crohn's disease (CD) is a chronic inflammatory condition of the gastrointestinal tract often requiring lifelong management. ${ }^{1}$ Although the prevalence of this disease is highest in Western countries, the incidence is rapidly rising in Asia. ${ }^{2}$ Azathioprine, a thiopurine analog, is a common agent for $\mathrm{CD}$ and has recently been reported to cause non-cirrhotic portal hypertension (NCPH). ${ }^{3}$ Although the pathogenic mechanism is not clear, the symptoms include those of portal hypertension, such as esophageal varices, thrombocytopenia, ascites, and splenomegaly, with normal hepatic function and no clear cause, such as liver cirrhosis. Some studies and case reports from Western countries have reported rare cases of idiopathic NCPH in pa-

Received January 29, 2020. Revised April 9, 2020. Accepted April 9, 2020. Correspondence to Eun Soo Kim, Department of Internal Medicine, School of Medicine, Kyungpook National University, 130 Dongdeok-ro, Jung-gu, Daegu 41944, Korea. Tel: +82-53-200-5879, Fax: +82-53-200-5879, E-mail: dandy813@hanmail.net tients with CD taking azathioprine. ${ }^{3-6}$ However, there were previously no Asian cases, which is a motive for publishing this report to aid in the management of CD in this region. Institutional Review Board of the Kyungpook National University Hospital waived the approval for this case because the patient identifying information was not included. Informed consent for this case report was obtained from the patient.

\section{CASE REPORT}

A 51-year-old male was admitted with a chief complaint of hematemesis. The patient had been taking mesalazine $2 \mathrm{~g}$ as a maintenance treatment since he was diagnosed with $\mathrm{CD}$ at other hospital 9 years ago. At diagnosis, the location and behavior of disease were ileal (L1) and non-stricturing, non-penetrating (B1), respectively. In 4 years after diagnosis, he visited our hospital and underwent a bowel resection surgery with small bowel-small bowel anastomosis due to a bowel perforation, and then he started azathioprine $50 \mathrm{mg}$ to prevent post- 
surgical relapse. At that time, there was no abnormal feature in a complete blood cell count (a white blood count of 4,620/ $\mathrm{mm}^{3}$, a hemoglobin of $12.8 \mathrm{~g} / \mathrm{dL}$, and a platelet of $385,000 / \mathrm{mm}^{3}$ ) and liver function test (aspartate aminotransferase [AST]/alanine aminotransferase [ALT] levels of 21/13 IU/L). Since then, however, the platelet count was gradually decreasing; 213,000 / $\mathrm{mm}^{3}$ in 6 months, $124,000 / \mathrm{mm}^{3}$ in 12 months, $105,000 / \mathrm{mm}^{3}$ in 18 months, and $102,000 / \mathrm{mm}^{3}$ in 24 months. After 3 years from azathioprine initiation, we decided to reduce the azathioprine dose from $50 \mathrm{mg}$ to $25 \mathrm{mg}$ because we found leukopenia $\left(3,390 / \mathrm{mm}^{3}\right)$ and thrombocytopenia $\left(83,000 / \mathrm{mm}^{3}\right)$. In 5 years after azathioprine use, the patient visited the department of emergency presenting with hematemesis over $200 \mathrm{~mL}$. The patient had a blood pressure of 126/86 $\mathrm{mmHg}$, a pulse of 120 beat per minute, a temperature of $36.5^{\circ} \mathrm{C}$, a respiratory rate of 20 breaths per minute, and no acute clinical presentation. The conjunctiva was not pale and jaundice was not observed in sclera. His lung and heart sounded normal. His abdominal bowel sound was normal. There was no tenderness at costovertebral angle and abdomen. Pitting edema at the lower extremity was not observed. The results of a complete blood cell count were as follows; a white blood count of $13,510 / \mathrm{mm}^{3}$, a hemoglobin of $9.5 \mathrm{~g} / \mathrm{dL}$, and a platelet of $199,000 / \mathrm{mm}^{3}$ (this normal platelet count might be due to hemoconcentration because it showed $76,000 / \mathrm{mm}^{3}$ after 2 days). The results of a liver function test were within a normal range showing AST/ ALT levels of 24/20 IU/L, an alkaline phosphatase level of 50 $\mathrm{IU} / \mathrm{L}$, and a gamma glutamyl transferase of $62 \mathrm{IU} / \mathrm{L}$. Other measurements were also not significant including a total protein level of $6.3 \mathrm{~g} / \mathrm{dL}$, an albumin level of $4.3 \mathrm{~g} / \mathrm{dL}$, and a total bilirubin level of $0.34 \mathrm{mg} / \mathrm{dL}$. The results of an evaluation for chronic liver disease were negative for hepatitis B surface antigen, hepatitis C virus antibody, antinuclear antibody, anti-mitochondrial antibodies, anti-smooth muscle antibody, and an-
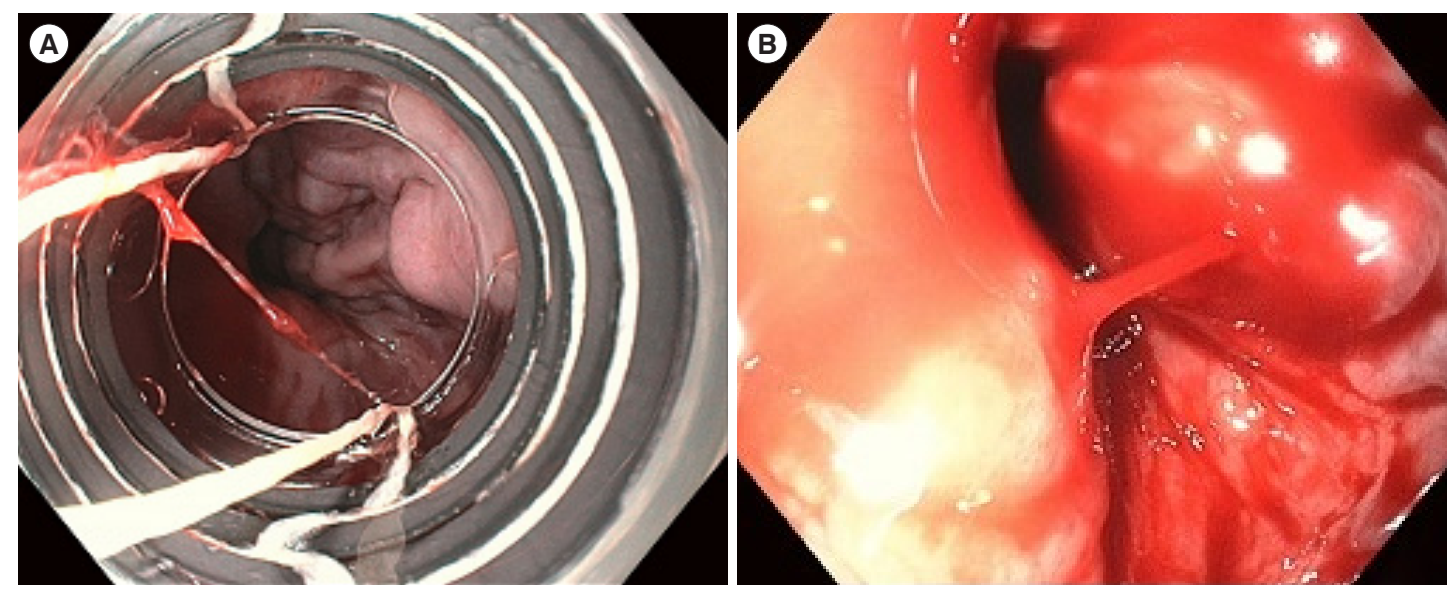

Fig. 1. Esophagogastroduodenoscopy findings. (A) Several bluish dilated vessels were noted at lower esophagus. (B) Spurting blood was noted at an esophageal varix.
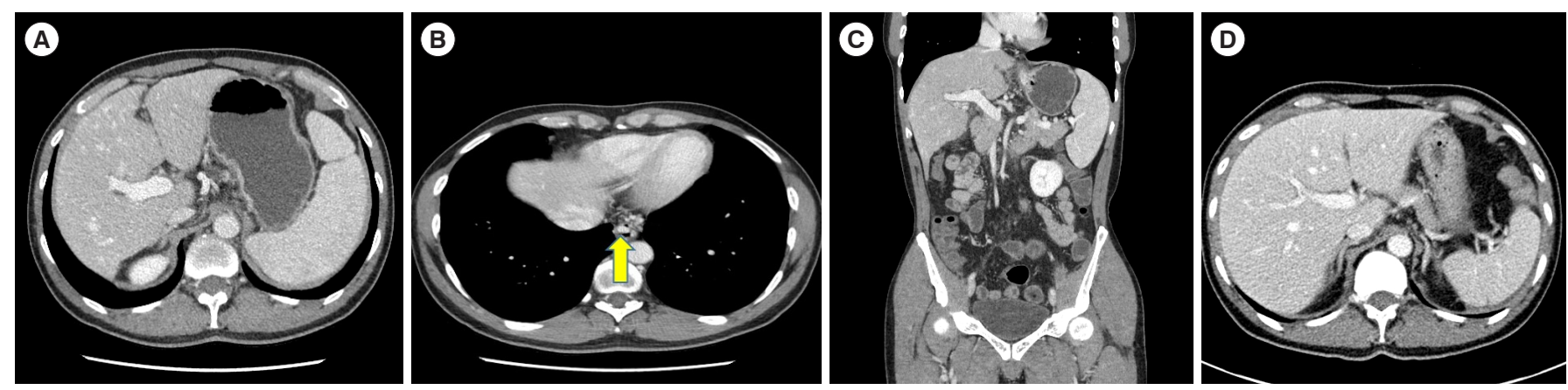

Fig. 2. Abdominal computed tomography (CT) images. $(A, B)$ Abdominal CT scan showed splenomegaly and dilated collateral vessels (arrow) in the lower esophagus. (C) There was no evidence of portal vein thrombosis at the coronal view of scan. (D) However, there was no evidence of splenomegaly on a previous image acquired 4 years ago at the time point of azathioprine initiation. 

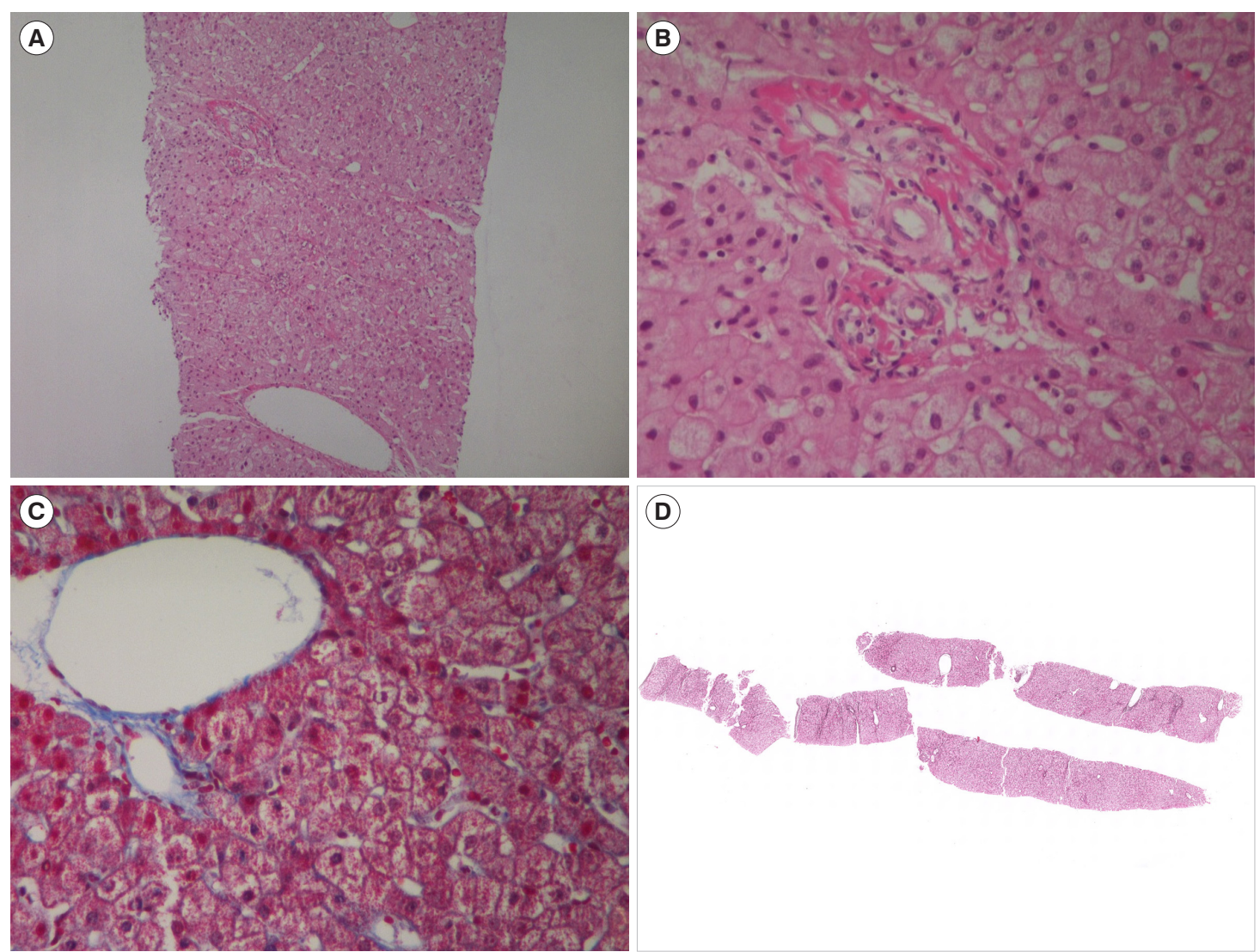

Fig. 3. Pathologic findings. ( $A, B)$ Liver biopsy showing no evidence of cirrhosis $(H \& E, A \times 100, B \times 400)$. (C) No fibrosis observed by trichrome staining $(\times 400)$. (D) No regenerative nodule was noted at the reticulin stain $(\times 20)$.

ti-liver kidney microsomal type 1 antibody. For evaluation of hematemesis, esophagogastroduodenoscopy was performed which showed several esophageal varices (Fig. 1A) and a focus of active bleeding from esophageal varices (Fig. 1B). Subsequently, endoscopic variceal ligation was successfully performed for bleeding management. Splenomegaly and dilated collateral vessels around distal esophagus were found by abdominal computed tomography (CT) on admission (Fig. 2A and $\mathrm{B}$ ), while the evidence of portal vein thrombosis, one of the main causes of portal hypertension, was not observed in the coronal view of CT scan (Fig. 2C). However, these features of portal hypertension had not been found by CT scans performed 4 years ago at the time of initiation of azathioprine (Fig. 2D). The stiffness measured with FibroScan test for liver cirrhosis was $7.6 \mathrm{kPa}$, which was below the range of liver cirrhosis. In a liver biopsy specimen, neither fibrosis nor evidence of hepatic cirrhosis, were observed (Fig. 3). Therefore, we concluded that NCPH was potentially related to azathioprine. After intensive discussion with the patient, azathioprine treatment was withdrawn, and biologic therapy was sched- uled when a relapse would occur during meticulous monitoring. A beta-blocker was added to prevent variceal bleeding due to portal hypertension. This patient has been continuing outpatient follow-up without any complications in 2 years thereafter.

\section{DISCUSSION}

The pathogenesis of $\mathrm{CD}$ is largely unknown and it is speculated that a combination of genetic, immunologic, and environmental factors may cause this disease, stimulating various approaches for the treatment of CD. In addition to conventional treatments including mesalazine, steroids and thiopurine derivatives, various biologic drugs and small molecules for CD therapy have emerged in recent years. ${ }^{1}$

Azathioprine and thiopurine analogs are agents used in organ transplantation, inflammatory bowel disease (IBD), autoimmune disease and malignancy. The hepatic toxicities of this drug mainly manifest as an increase in the transaminase level in liver function tests. Most of these toxicities are mild and 
generally relieved by reducing the dose.

In rare cases, however, thiopurine treatment may cause nodular regenerative hyperplasia (NRH) of the liver, and the early detection of NRH is difficult because of the lack of symptoms in early stage and the absence of diagnostic markers. ${ }^{6,7}$ The diagnosis of NRH is made by liver biopsy with reticulin stain and is often difficult because regenerative nodules may be missed with needle biopsy. ${ }^{8}$ This may explain the reason why we could not observe the definite evidence of NRH in the microscopic evaluation of the patient's liver with reticulin stain (Fig. 3D). Vernier-Massouille et al. ${ }^{9}$ tracked the development of NRH in patients with IBD taking azathioprine and reported incidence rates of $0.5 \%$ and $1.25 \%$ over 5 years and 10 years, respectively. Many diseases and agents are known to be involved in the development of this condition and the use of azathioprine or thioguanine were reported to be one of the risk factors. ${ }^{10}$

Despite unknown etiology of NRH, Musumba ${ }^{11}$ showed that the mechanisms of NRH in patients with IBD include immunological and thrombotic factors in addition to external factors such as azathioprine. The uncontrolled inflammatory response itself in patients with IBD stimulates these factors, which also could cause NRH. However, the injury to endothelial cells of the sinusoids and hepatic veins caused by non-thrombotic occlusion of vessels due to depletion of glutathione induced by azathioprine therapy, ${ }^{12}$ may be attributed to hypoperfusion of liver parenchyma and parenchymal atrophy leading to subsequent development of fibrosis and portal hypertension. ${ }^{13}$ This event result in alternating areas of hepatocyte atrophy and adaptive hepatocyte hyperplasia. ${ }^{11}$ This response is known to develop from 3 months to 3 years after initiating thiopurine treatment. $^{12}$

However, apart from NRH, there were many conditions or terms describing the histological alterations observed in patients with portal hypertension in absence of cirrhosis, increasing the confusion. ${ }^{4}$ Therefore, in 2011, a group of European experts in portal hypertension proposed the term idiopathic NCPH replacing hepatoportal sclerosis, idiopathic portal hypertension, incomplete septal cirrhosis, and NRH. ${ }^{14}$ Since then, the term of idiopathic NCPH has been used in the literature related with thiopurine in IBD patients. Suárez Ferrer et al. ${ }^{12}$ reported the prevalence of idiopathic NCPH as $0.43 \%$ in patients with CD taking a thiopurine, suggesting that this complication should be considered during the treatment of IBD despite scarcity.

This disease is diagnosed when symptoms of portal hypertension, such as esophageal varix, thrombocytopenia, ascites, and splenomegaly, manifest with normal hepatic function and no clear cause such as liver cirrhosis. ${ }^{4,13,15,16}$ The possible causes of idiopathic NCPH include autoimmune disease, chronic infection, genetic disease, coagulation factor disease, and agents such as azathioprine. The course may progress to ascites, hepatic failure, and even portal vein thrombosis. ${ }^{17}$ The treatment goals for idiopathic NCPH are prevention and treatment of bleeding esophageal varices and symptoms of other complications. ${ }^{17}$

Seiderer et al. ${ }^{18}$ reported that stopping azathioprine treatment improved the symptoms of portal hypertension, while Pola et al. ${ }^{3}$ reported that NRH and hepatoportal sclerosis caused by 6-mercaptopurine were irreversible. Given that there is no consensus on the guidelines for the treatment of NCPH, stopping causative agents like azathioprine and treating manifested symptoms may be a possible approach for recovery.

In conclusion, we report the first Asian case of idiopathic NCPH possibly related to the long-term use of azathioprine for the treatment of CD. Liver monitoring might be needed for this rare hepatic complication of thiopurine treatment. When thrombocytopenia and splenomegaly occur in patients with IBD taking a thiopurine drug for more than 3 months, idiopathic NCPH should be considered for the list of possible diagnosis.

\section{ADDITIONAL INFORMATION}

\section{Funding Source}

The authors received no financial support for the research, authorship, and/or publication of this article.

\section{Conflict of Interest}

No potential conflict of interest relevant to this article was reported.

\section{Author Contribution}

Conceptualization: Kim ES. Visualization: Kim ES, Han MH. Writing - original draft: Seo JW. Writing - review and editing: Kim ES, Kweon YO. Approval of final manuscript: all authors.

\section{ORCID}

Seo JW

Kim ES

Han MH

Kweon YO https://orcid.org/0000-0001-7668-0995 https://orcid.org/0000-0003-0806-9136 https://orcid.org/0000-0001-8856-553X https://orcid.org/0000-0001-5708-7985 


\section{REFERENCES}

1. Torres J, Mehandru S, Colombel JF, Peyrin-Biroulet L. Crohn's disease. Lancet 2017;389:1741-1755.

2. Kaplan GG, Ng SC. Understanding and preventing the global increase of inflammatory bowel disease. Gastroenterology 2017;152:313-321.

3. Pola S, Boland BS, Lin GY, Kuo A, Sandborn WJ. Non-cirrhotic portal hypertension due to 6-mercaptopurine use for Crohn's disease. Dig Dis Sci 2014;59:1642-1644.

4. Riggio O, Gioia S, Pentassuglio I, Nicoletti V, Valente M, d’Amati G. Idiopathic noncirrhotic portal hypertension: current perspectives. Hepat Med 2016;8:81-88.

5. Dooremont D, Decaestecker J, De Wulf D, et al. Azathioprine induced serious portal hypertension: a case series of three IBD patients and review of the literature. Acta Gastroenterol Belg 2013;76:342-346.

6. Cohen-Ezra O, Avni Y, Morgenstern S, Ben-Ari Z. Nodular regenerative hyperplasia as a complication of thiopurine treatment in a patient with inflammatory bowel disease. Harefuah 2012;151:675-721.

7. Simsek M, Meijer B, Ramsoekh D, et al. Clinical course of nodular regenerative hyperplasia in thiopurine treated inflammatory bowel disease patients. Clin Gastroenterol Hepatol 2019; $17: 568-570$

8. Reshamwala PA, Kleiner DE, Heller T. Nodular regenerative hyperplasia: not all nodules are created equal. Hepatology 2006;44:7-14.

9. Vernier-Massouille G, Cosnes J, Lemann M, et al. Nodular re- generative hyperplasia in patients with inflammatory bowel disease treated with azathioprine. Gut 2007;56:1404-1409.

10. Simsek M, Seinen ML, de Boer NKH. Nodular regenerative hyperplasia in inflammatory bowel disease patients with allopurinol-thiopurine cotherapy. Eur J Gastroenterol Hepatol 2018;30:1254-1255.

11. Musumba CO. Review article: the association between nodular regenerative hyperplasia, inflammatory bowel disease and thiopurine therapy. Aliment Pharmacol Ther 2013;38:10251037.

12. Suárez Ferrer C, Llop Herrera E, Calvo Moya M, et al. Idiopathic portal hypertension regarding thiopurine treatment in patients with inflammatory bowel disease. Rev Esp Enferm Dig 2016;108:79-83.

13. Fiel MI, Schiano TD. Idiopathic noncirrhotic portal hypertension. Semin Diagn Pathol 2019;36:395-403.

14. Schouten JN, Garcia-Pagan JC, Valla DC, Janssen HL. Idiopathic noncirrhotic portal hypertension. Hepatology 2011;54:10711081.

15. Hernández-Gea V, Baiges A, Turon F, Garcia-Pagán JC. Idiopathic portal hypertension. Hepatology 2018;68:2413-2423.

16. Sarin SK, Khanna R. Non-cirrhotic portal hypertension. Clin Liver Dis 2014;18:451-476.

17. Guido M, Sarcognato S, Sacchi D, Colloredo G. Pathology of idiopathic non-cirrhotic portal hypertension. Virchows Arch 2018;473:23-31.

18. Seiderer J, Zech CJ, Diebold J, et al. Nodular regenerative hyperplasia: a reversible entity associated with azathioprine therapy. Eur J Gastroenterol Hepatol 2006;18:553-555. 